\title{
A Multi-Axis Biodynamic Measuring Handle for a Human Hand-Arm System
}

\author{
Luka Knez - Janko Slavič* - Miha Boltežar \\ University of Ljubljana, Faculty of Mechanical Engineering, Slovenia
}

The biodynamic response method is increasingly being used to study the human hand-arm system and vibration-induced injuries that affect the hand. Most measurements are made in the dominant forearm direction of excitation, but recently research has turned to multi-axis measurements as well as excitation. This study looks at a new instrument handle that measures the biodynamic responses at the palm and the fingers in multiple directions and, at the same time, removes the need to stop the test and change the orientation of the handle. In addition to the biodynamic response, the sensors inside the handle are able to measure the static push and grip forces simultaneously and therefore remove the need for an additional force plate or force sensor at the handle base, which is typically used to measure the push force. The apparent mass of the handle was measured in order to determine the usable frequency range of the system. Additionally, the apparent mass distribution along the hand was investigated and it was found that the apparent mass distribution along the hand varies with frequency. Keywords: biodynamic response, human hand-arm system, multi-axis measurement, apparent mass distribution, instrument handle

\section{O INTRODUCTION}

For health reasons, a great deal of research has been done in the field of vibration transmissibility from the excitation source to the user's hands [1]. The measurement techniques that are most frequently used are the ISO 10819 [2] standardized method and - as an alternative to the standardized method - the driving-point, biodynamic-response method [3] and [4].

The standardized ISO 10819 method measures the vibration transmissibility to the hands when the user wears anti-vibration gloves. The method uses a special adapter, which contains a miniature accelerometer that is held in the palm of the hand. The operator holds a vibrating handle, which then simulates the hand-tool excitations. To keep the measurements as realistic as possible, the grip and push forces are also measured and it is the operator's task to keep them within the prescribed limits. The grip force is measured at the handle itself and the push force is usually measured at the handle-shaker interface or with a force plate located under the operator. The vibration transmissibility to the hand is derived from accelerations measured at the adapter and at the reference point in the handle.

However the adapter can cause measurement errors; therefore, other methods have been developed. One of the recent and most promising methods used for determining the vibration transmissibilities and the dynamic properties of the hand is the biodynamicresponse method, researched among others by [3] to [8]. The method requires simultaneous measurements of the dynamic motion and forces at the handhandle interface and eliminates the need for a palm adapter. A comparison between the standardized and biodynamic methods made by Dong et al. [6] shows that the biodynamic method performs better than the standardized method. The method has also been improved to measure the biodynamic responses separately at the palm and at the fingers [3] and [9].

There are various types of vibration sources that excite the hands in multiple directions; the ISO 5349-1 [10] and ISO10068 standards [11] denote those directions as the $X_{\mathrm{h}^{-}}, Y_{\mathrm{h}^{-}}$and $Z_{\mathrm{h}}$-axis. The forearm direction or $Z_{\mathrm{h}}$-axis is the dominant direction in the operation of several tools [12] and [13]; however, there are certain tools that excite strongly in more axes (e.g., chain-saw, hand grinder, etc. [13]). To protect the user from the hazards of using such tools, research must be conducted for multiple axes. Recently, Dong et al. [14] carried out a multi-axis study where the system used can excite and measure the hand responses simultaneously in all three orthogonal directions. The researched approach requires the simultaneous use of three shakers and, to measure the fingers after the palm measurements are completed, the test needs to be stopped and the handle rotated by 180 degrees.

This study researches a measuring system that is used for the biodynamic response measurements of the hand-arm system (HAS) at the palm and at the fingers for all three directions. The design of the system enables concurrent measurements of both the palm and the fingers, therefore removing the need to rotate the handle. The same sensors that are used for the dynamic response measurements are also used for the measurement of the static grip and push forces. Since both static forces are measured in the handle itself, the force plate, which is commonly used to measure the push force, is no longer necessary. 
The hand is a complex system of tissues, muscles and bones, and its parts differ in terms of mass and strength. As a result, some parts of the hand might be more susceptible to vibrations than others. The distribution of the apparent mass along the hand was, therefore, examined in an effort to clarify this.

The manuscript is organized as follows. The basics of biodynamic theory are presented in Section 1. Section 2 covers the instrumental handle and its dynamic characterization, the measuring setup and some details about multi-axis signal acquisition and processing. The results of the apparent mass of the HAS are shown in Section 3 for the $Z_{\mathrm{h}}$ - and $Y_{\mathrm{h}}$-axis of excitation and the distribution of the apparent mass along the hand is also presented.

\section{THEORETICAL BACKGROUND}

The theory used to derive the biodynamic response of the HAS is only briefly presented here; it is explained in detail by Dong et al. [3]. A biodynamic system is characterised by the apparent mass (AM), the mechanical impedance (MI), and the apparent stiffness (AS). In this study only the AM will be used, which is defined as:

$$
A M=\frac{\tilde{F}}{\tilde{A}},
$$

where $\tilde{F}$ is the dynamic force of the hand, and $\tilde{A}$ is the dynamic acceleration at the hand-handle interface. These parameters must be measured simultaneously in the direction of excitation. The reader should note that the MI and the AS can be computed from the AM data.

In the frequency domain, the biodynamicresponse parameters can be computed from:

$$
Z(w)=\frac{G_{f m}(w)}{G_{m m}(w)^{\prime}},
$$

where $Z(\omega)$ represents any of the biodynamic-response parameters, $G_{f m}$ is the cross-spectrum of the force and the dynamic motion (acceleration for the AM, velocity for the MI or displacement for the AS), and $G_{m m}$ is the auto-spectrum of the dynamic motion. The results obtained are mathematically complex:

$$
Z(\omega)=Z_{R e}(\omega)+Z_{I m}(\omega) \cdot j
$$

where $Z_{R e}(\omega)$ is the real component of the result, $Z_{l m}(\omega)$ is the imaginary component and $j=\sqrt{-1}$.

The measured dynamic force $\tilde{F}$ is a combination of the biodynamic force and the inertial force of the handle. For the biodynamic response of the hand $Z_{\text {Hand }}$ it is therefore necessary to deduct the effect of the measuring handle $Z_{\text {Handle }}$ from the total (handle and hand) biodynamic response $Z_{\text {TotalHand: }}$ :

$$
Z_{\text {Hand }}(\omega)=Z_{\text {TotalHand }}(\omega)-Z_{\text {Handle }}(\omega) \text {. }
$$

For the biodynamic response of the palm or the fingers the biodynamic parameters must be measured separately at the palm and at the fingers. The AM at the fingers $A M_{\text {Fingers }}$ is therefore computed as:

$$
\begin{gathered}
A M_{\text {Fingers }}(\omega)= \\
=A M_{\text {TotalFingers }}(\omega)-A M_{\text {HandleFingers }}(\omega),
\end{gathered}
$$

where $A M_{\text {TotalFingers }}$ is the total $\mathrm{AM}$ (with the human hand on the handle) and $A M_{\text {HandleFingers }}$ is the AM of the handle measured (without the human hand on the handle) at the fingers' side of the handle. A similar procedure is applied for the biodynamic response of the palm. If necessary, the biodynamic response of the gloved hand can also be evaluated, see, for example, Dong et al. [3] for details.

\section{APPARATUS}

\subsection{The Measuring Handle}

A system capable of measuring in all three axes $\left(X_{\mathrm{h}}\right.$, $Y_{\mathrm{h}}$ and $Z_{\mathrm{h}}$ ) has been developed [15] and is based on the research of Dong et al. [6] and [3] and Shibata et al. [4]. A measuring handle, with a diameter of $40 \mathrm{~mm}$ and a length of $110 \mathrm{~mm}$, was made as shown in Fig. 1. The handle consists of an upper and lower stainlesssteel cover, each with a 3 -axis accelerometer attached. The upper cover is used for the palm and the lower for the finger measurements. Each cover is screwed onto two 3-axis force sensors and the force sensors are screwed onto an aluminum frame. A circular plate is welded onto the bottom of the aluminum frame where it can be fixed onto an electrodynamic shaker.

The 3-axis force sensors, Kistler 9317B type, are used to measure the biodynamic forces in all three directions. The vector forces shown in Fig. 1 are labelled as $\mathbf{P}_{1}$ and $\mathbf{P}_{2}$ for the palm side and $\mathbf{F}_{1}$ and $\mathbf{F}_{2}$ for the finger side. These vector forces are measured in the hand's coordinate system shown in Fig. 1. The piezoelectric force transducers together with the Kistler 5073 charge amplifiers can measure the dynamic, as well as the quasi-static grip and push forces. Therefore, every force signal measured by the sensors contains dynamic as well as static components of the forces, which are obtained by appropriate 

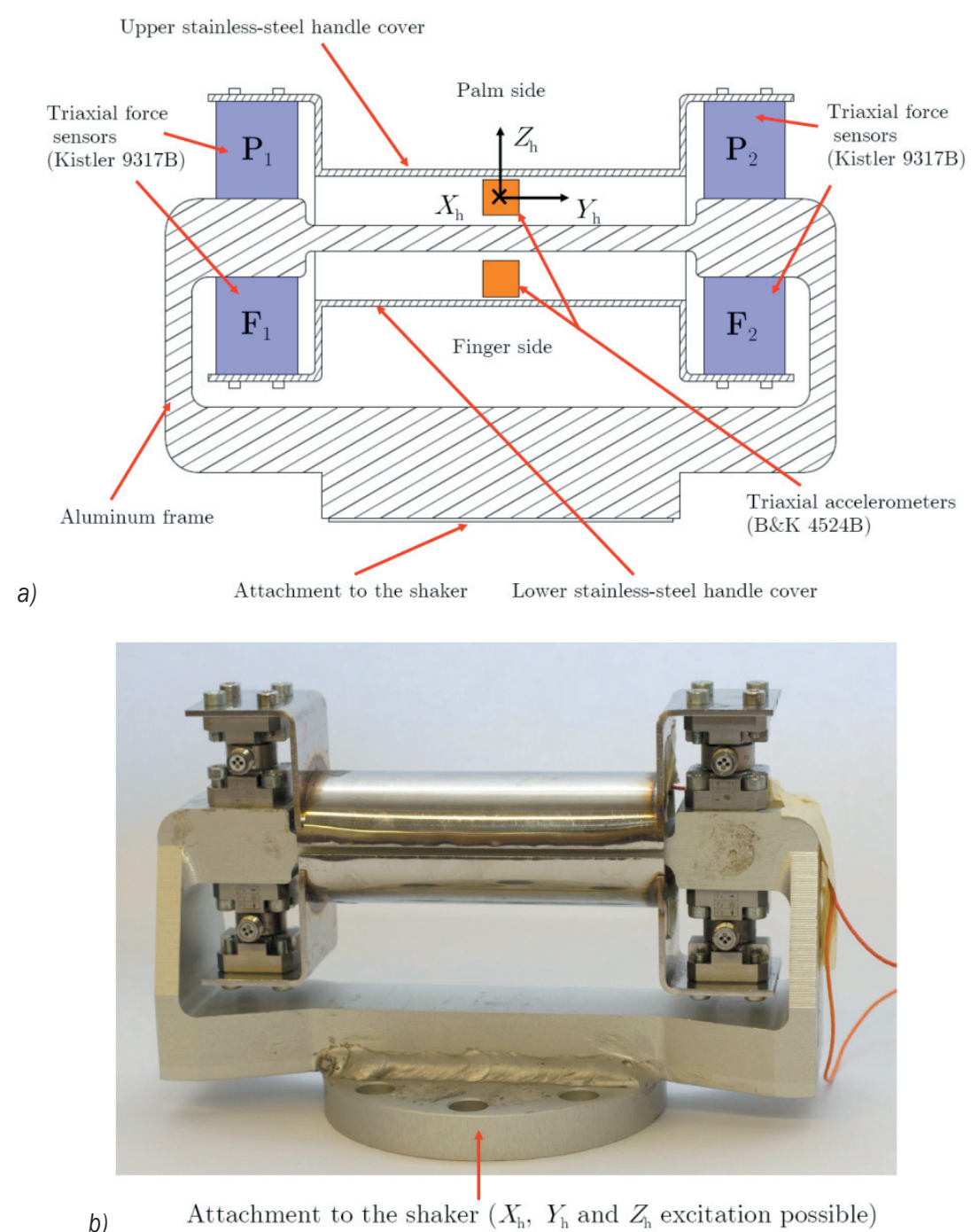

Fig. 1. The measuring handle: a) a sketch of the handle; b) a photograph of the handle

signal processing (see Sections 2.2 and 2.3). The selected charge amplifier also has a low zero-drift, which is approximately $0.25 \mathrm{~N} / \mathrm{min}$. To measure the dynamic accelerations in all three directions, two 3-axis accelerometers, Brüel \& Kjaer 4524B type, are used. The vector accelerations are denoted as $\mathbf{A}_{\mathbf{P}}$ and $\mathbf{A}_{\mathbf{F}}$ for the palm and finger sides, respectively. The accelerometers are attached to the middle of each cover, as suggested by Adewusi et al. [16].

\subsection{Identifying the Apparent Mass}

The details of the signal processing of the measured forces and accelerations are shown in Fig. 2. To obtain the force at the fingers, the forces $\mathbf{F}_{1}$ and $\mathbf{F}_{2}$ are first summed:

$$
\mathbf{F}=\mathbf{F}_{1}+\mathbf{F}_{2}
$$

This summation was made for each segment (2500 samples per segment, resulting in $0.25 \mathrm{~s}$ ). The sampling frequency was $10-\mathrm{kHz}$ and a low-pass filter of $20-\mathrm{kHz}$ was used. The force at the fingers $\mathbf{F}$ and the acceleration vector at the finger side $\mathbf{A}_{\mathbf{F}}$ are transformed into the frequency domain. The Hamming window was used and the 2500 samples resulted in a 4-Hz frequency resolution.

If the AM with the human hand on the handle (specifically the fingers) is measured, then the subscript "TotalFingers" is used; if the handleonly (finger side) is measured, the subscript "HandleFingers" is used. As discussed later, measurements with the hand on and without the 


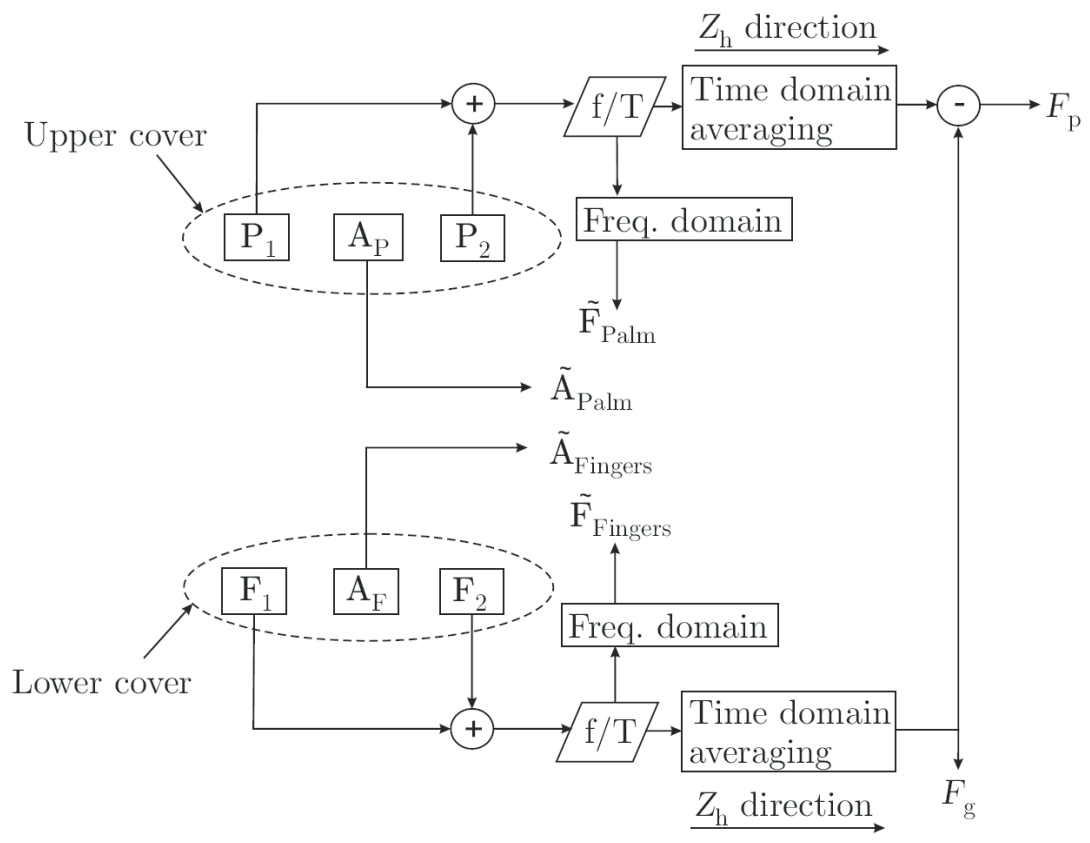

Fig. 2. Flowchart of the dynamic forces $\tilde{F}$ and the accelerations $\tilde{A}$ and the quasi-static grip $F_{\mathrm{g}}$ and push forces $F_{p}$

hand on the handle are required. Here, the hand-onhandle equations will be discussed in detail, but a similar approach is used for the handle only. The frequency-domain forces and accelerations when the hand is on the handle are denoted as $\tilde{\boldsymbol{F}}_{\text {TotalFingers }}$ and $\tilde{\boldsymbol{A}}_{\text {TotalFingers }}$, and the AM per segment $i$ is obtained as:

$$
\boldsymbol{A} \boldsymbol{M}_{\text {TotalFingers, } i}=\frac{\tilde{\boldsymbol{F}}_{\text {TotalFingers }}}{\tilde{\boldsymbol{A}}_{\text {TotalFingers }}},
$$

where the division needs to be performed for each $X_{\mathrm{h}}$, $Y_{\mathrm{h}}$ and $Z_{\mathrm{h}}$ axis separately (at the element level).

The AM of the fingers on the handle is obtained by segment averaging (100 averages have been used, where the segments overlapped at $50 \%$ ):

$$
\boldsymbol{A M}_{\text {TotalFingers }}=\frac{1}{100} \sum_{i=1}^{100} A M_{\text {TotalFingers }, i} \text {. }
$$

Finally, as discussed in the theoretical section, from measurements of the handle only and the handle with the hand, the biodynamic response of the fingers can be identified from Eq. (4).

For the biodynamic response of the palm, the palm-side apparent mass $A M_{\text {Palm }}$ is required. A similar approach to the finger side is used, but it is based on the time-domain summation at the palm side:

$$
\boldsymbol{P}=\boldsymbol{P}_{1}+\boldsymbol{P}_{2}
$$

and time-domain acceleration $\boldsymbol{A}_{P}$.

\subsection{Identifying the Push and Grip Forces}

In addition to the dynamic forces, the static push and grip forces, as defined in ISO 10819 , need to be measured. As these forces are always in the $Z_{\mathrm{h}}$-axis of the hand, only the forces in this particular direction need to be processed. As can be seen in Fig. 2, the grip force $\mathrm{Fg}$ is the summation of the forces $F_{1, Z \mathrm{~h}}$ and $F_{2, \mathrm{Zh}}$ ( $Z_{\mathrm{h}}$ is used to denote the component of the vector in the $Z_{\mathrm{h}}$ direction). To obtain the static component, the measured samples are averaged in the time-domain (the averaging period was $0.25 \mathrm{~s}$, which corresponds to 2500 measured samples):

$$
F_{g}=\operatorname{Mean}\left(F_{1, Z h}+F_{2, Z h}\right) \text {. }
$$

Similarly, the push force $F_{p}$ is obtained:

$$
F_{p}=\operatorname{Mean}\left(P_{1, Z h}+P_{2, Z h}-\left(F_{1, Z h}+F_{2, Z h}\right)\right) \text {. }
$$

\subsection{Identifying the Apparent Mass Distribution at the Fingers and the Palm}

This study also looks at the AM distribution along the hand. For this reason, four hand regions have been defined (Fig. 3): the upper finger side (thumb, index and middle finger), the lower finger side (pinky and 
ring finger), the upper palm side, and the lower palm side.

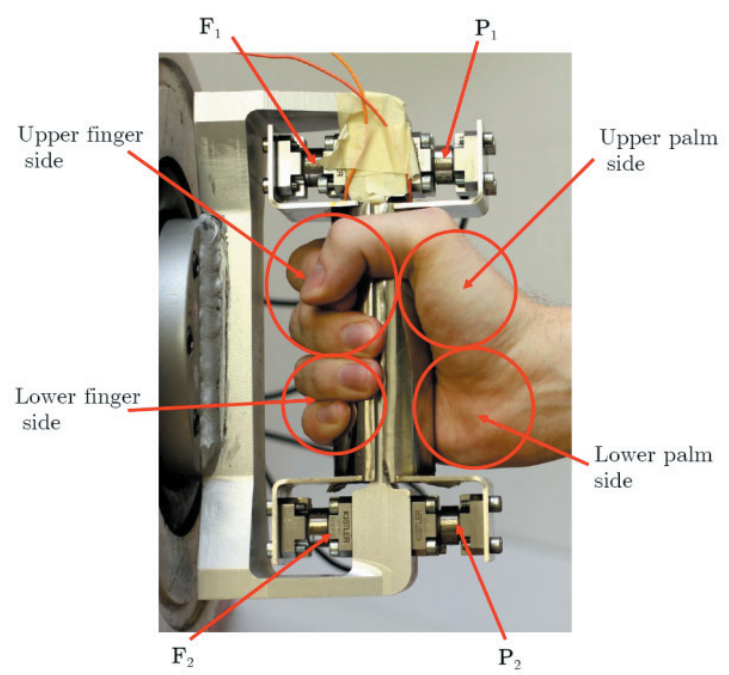

Fig. 3. The hand gripping the handle, divided into four areas, measured with four force sensors

The AM distribution at the finger side is estimated by:

$$
\boldsymbol{R}_{\text {Fingers }}=\frac{\boldsymbol{A} \boldsymbol{M}_{1, \text { Fingers }}}{\boldsymbol{A} \boldsymbol{M}_{2, \text { Fingers }}},
$$

where $\boldsymbol{A} \boldsymbol{M}_{j, \text { Fingers }}$ are the $\mathrm{AM}$ vectors at the upper $(j=1)$ and lower $(j=2)$ finger sides, respectively. The division in Eq. (12) needs to be performed for each $X_{\mathrm{h}}$, $Y_{\mathrm{h}}$ and $Z_{\mathrm{h}}$ axis separately (at the element level).

The AM vectors $\boldsymbol{A M}_{j, \text { Fingers }}$ are obtained by subtracting the AM measured at the handle only, $\boldsymbol{A M}_{j, \text { HandleFingers, }}$, from the AM measured when the hand is on the handle, $\boldsymbol{A} \boldsymbol{M}_{j, \text { TotalFingers: }}$ :

$$
\boldsymbol{A M}_{j, \text { Fingers }}=\boldsymbol{A} \boldsymbol{M}_{j, \text { TotalFingers }}-\boldsymbol{A} \boldsymbol{M}_{j, \text { HandleFingers }} \text {. }
$$

The AM values are evaluated similarly for each direction as in Eq. (8), except that the forces $\tilde{\boldsymbol{F}}_{1}$ and $\tilde{\boldsymbol{F}}_{2}$ are not summed but used separately, i.e.:

$$
\boldsymbol{A M}_{j, \text { TotalFingers }}=\frac{\tilde{\boldsymbol{F}}_{j, \text { TotalFingers }}}{\tilde{\boldsymbol{A}}_{F, \text { TotalFingers }}},
$$

is the AM when the hand is on the handle.

The dynamic forces $\tilde{\boldsymbol{F}}_{j}$ are obtained from the $\boldsymbol{F}_{j}$ by frequency-domain averaging and the procedure is similar to that described in Section 2.2, except that 5000 samples were used and resulted in a $2-\mathrm{Hz}$ resolution.
The force exerted by any part of the hand is distributed to both of the force sensors; however, the change in the dynamic response is dependent on the position of the hand-part with regards to the handle. The apparent mass calculated from the forces $\tilde{\boldsymbol{F}}_{j}$ therefore gives additional information on the dynamics of the hand, specifically which part of the hand exerts more force on the handle.

Similarly, the dynamic acceleration $\tilde{A}_{\text {TotalFingers }}$ is obtained from $\boldsymbol{A}_{F}$. A single accelerometer is used for both the upper and lower sides since the frequency range of interest $(10$ to $500 \mathrm{~Hz})$ is sufficiently below the first natural frequency of the system $(550 \mathrm{~Hz})$. Rigid motion of the handle cover can therefore be assumed and the measured acceleration is equal for both sides of the hand $(j=1,2)$. The differences compared to the apparent mass are measured via the dynamic forces.

A similar approach is used for the palm AM distribution:

$$
\boldsymbol{R}_{\text {Palm }}=\frac{\boldsymbol{A} \boldsymbol{M}_{1, \text { Palm }}}{\boldsymbol{A} \boldsymbol{M}_{2, \text { Palm }}}
$$

\subsection{Experimental Setup}

The experimental setups used in this study are illustrated in Fig. 4. For the $Z_{\mathrm{h}}$-axis measurements the handle was fixed on an LDS V555 electrodynamic shaker that was tilted in the appropriate direction. For the $Y_{\mathrm{h}}$-axis measurements a larger LDS V875 electrodynamic shaker was used. With a proper experimental setup the biodynamic responses in the $\mathrm{Xh}$ direction could also be measured.

During the measurements the handle was subjected to a broadband random vibration with a power spectral density (PSD) of $5\left(\mathrm{~m} / \mathrm{s}^{2}\right)^{2} / \mathrm{Hz}$ in the frequency range 10 to $500 \mathrm{~Hz}$.

\subsection{Dynamic Characterization of the Handle}

The instrument handle is a dynamical system and as such has its own dynamic properties, which are determined using modal testing [17] to [19]. For proper results, the measurement system's natural response should be well outside the measurement range. For this reason the AM of the handle was measured for the $Z_{\mathrm{h}}$ and $Y_{\mathrm{h}}$ directions of the excitation in order to determine the usable frequency range. Fig. 5 presents the AM at the palm and Fig. 6, the AM at the fingers. 


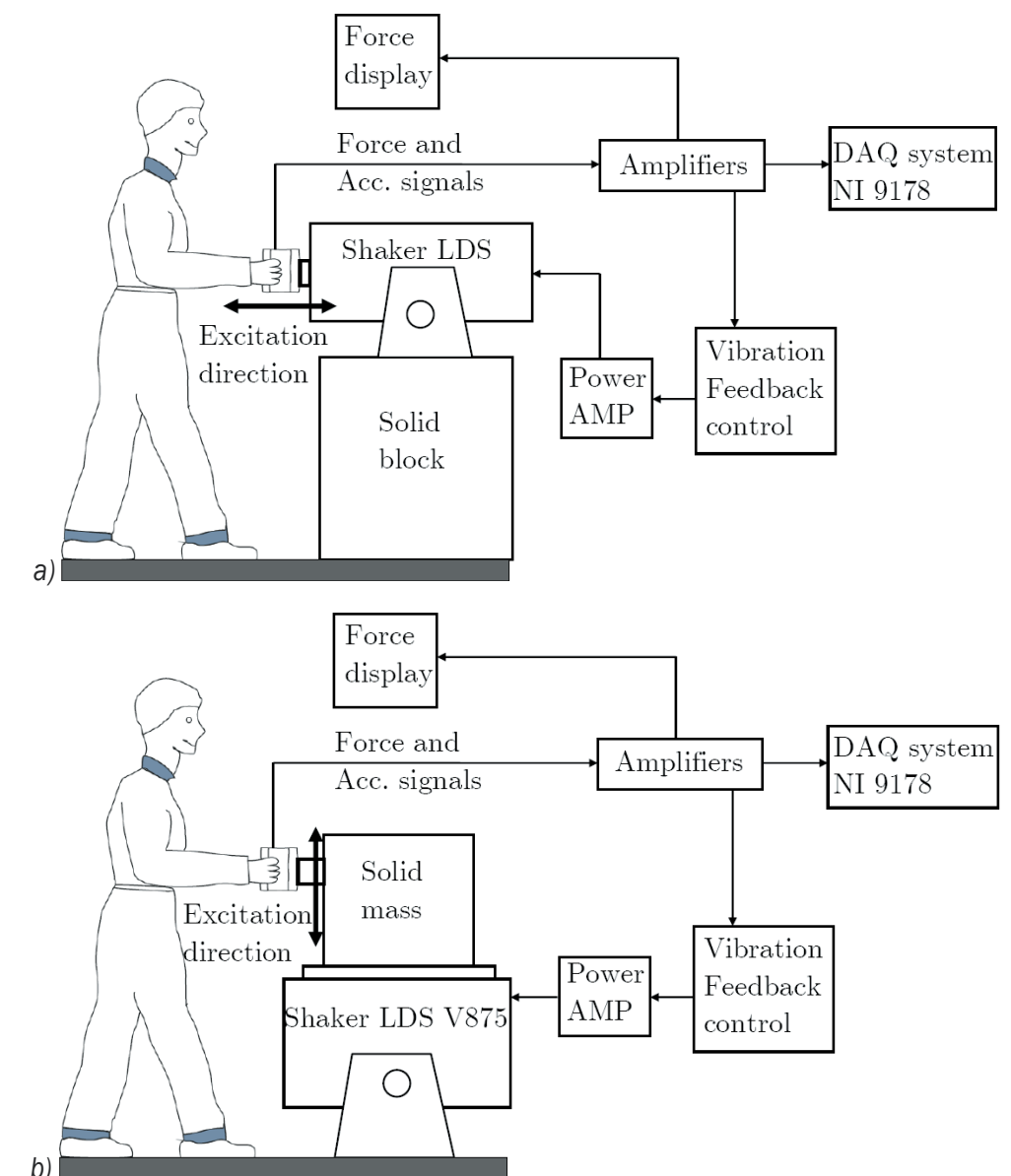

Fig. 4. Experimental setup for excitation in the: a) $Z_{h}$ direction; b) $Y_{h}$ direction

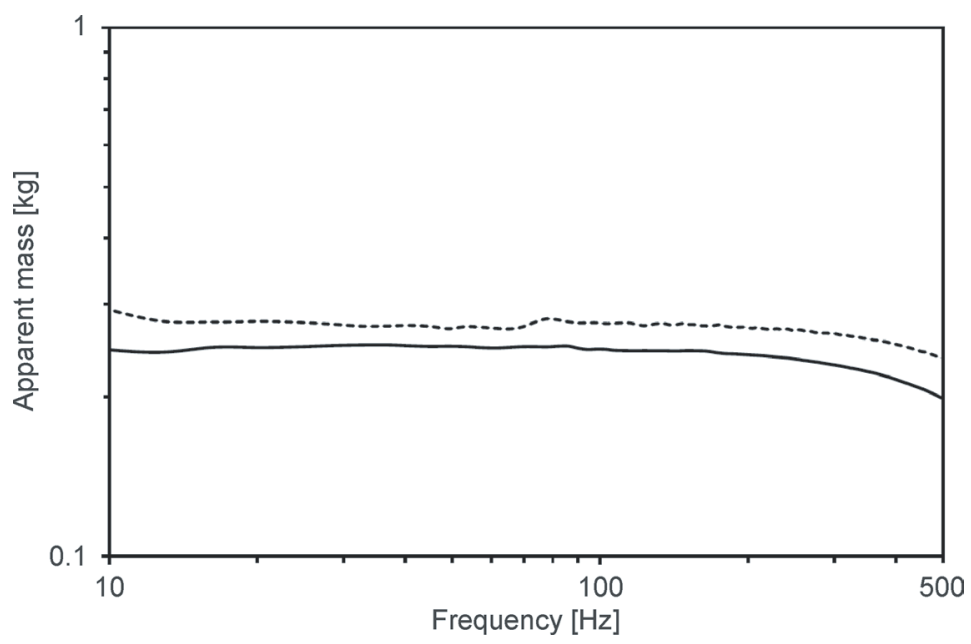

Fig. 5. Apparent mass response of the handle measured at the palm (- $Z_{\mathrm{h}}$ direction; --- $Y_{\mathrm{h}}$ direction)

There are slight differences between the response of the handle for the $Z_{\mathrm{h}}$ - and $Y_{\mathrm{h}}$ - axis. The reason for the differences was found in the structural dynamics of the measuring system, because the handle's dynamic properties are dependent on the direction of the excitation. The fundamental resonant frequency of the handle in the $Z_{\mathrm{h}}$-axis was measured at $572 \mathrm{~Hz}$, while in the $Y_{\mathrm{h}}$-axis it was found at $550 \mathrm{~Hz}$. Although the rigidity of the handle was assumed, the peak in the $Y_{\mathrm{h}}$-axis has a larger influence on the measurements 


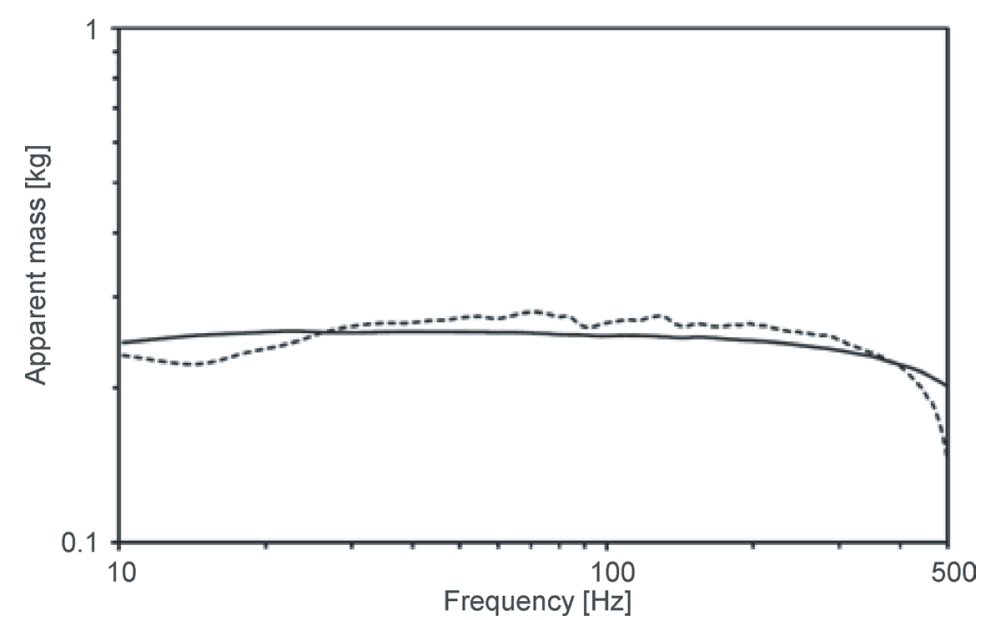

Fig. 6. Apparent mass response of the handle measured at the fingers $\left(-Z_{\mathrm{h}}\right.$ direction; - - - $Y_{\mathrm{h}}$ direction)

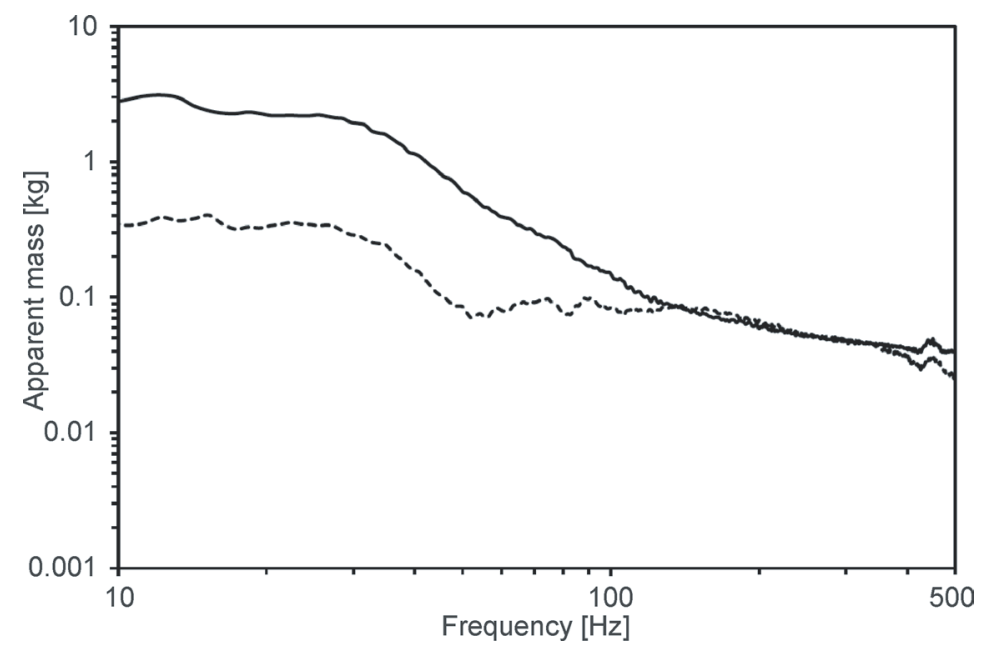

Fig. 7. Apparent mass of the subject for $Z_{h}$-axis excitation (- palm; - - - fingers)

than the one in the $Z_{\mathrm{h}}$-axis. This results in a slightly different apparent mass of the handle.

\section{RESULTS AND DISCUSSION}

The results of the measuring system are presented in this section - specifically the AM of the HAS - and a comparison with previous research is made. Only one subject was used, since the focus of the research is on the handle itself and not on inter- and intra-subject differences. The experimental procedure is based on the procedure described in the ISO 10819 standard and Dong et al. [3], and is the same for both directions of excitation. The research on posture by Aldien et al. [20] and Adewusi et al. [21] was also considered.

The biodynamic response of the handle alone was measured first and afterwards one subject held the handle with his bare hand. The subject was given all the relevant instructions prior to the measurements. The grip force was $30 \mathrm{~N}$ and the push force was $50 \mathrm{~N}$ throughout the measurement. Both forces were constantly displayed on a monitor, as indicated in Fig. 8.

The AM was identified using Eq. (8). The subject's AM was measured three times for each direction of the excitation and the results were then averaged to minimize the inter-subject differences. The measurement frequency range chosen was from 10 to $500 \mathrm{~Hz}$, in accordance with the excitation subjected to the handle, see Section 2.

\subsection{The Biodynamic Response of the HAS in the $Z_{h}$ - and $\mathrm{Y}_{\mathrm{h}}$-axis}

The measurements in the dominant $Z_{\mathrm{h}}$-axis were made first. Fig. 7 shows the resulting AM of one subject 
measured at the palm and at the fingers. The results are comparable to those reported by Dong et al. [6] and Concettoni and Griffin [8].

The values of the amplitude measured at the palm agree well with the one reported by Dong et al. [6]. Both results show a nearly constant magnitude of $1.1 \mathrm{~kg}$ from 10 to $30 \mathrm{~Hz}$ and a resonance peak was observed in both studies at around $450 \mathrm{~Hz}$ with a magnitude of $0.4 \mathrm{~kg}$.

Concettoni and Griffin [8] measured the apparent mass of a hand pushing down on a vibrating plate with different coupling conditions. Their study found two frequency ranges where finger resonances are expected (10 to $16 \mathrm{~Hz}$ and 50 to $90 \mathrm{~Hz}$ ), depending on the dynamic properties of the measured subject. The data presented in this study agrees with their findings, with a peak at $16 \mathrm{~Hz}$ and two more at 75 and $90 \mathrm{~Hz}$.

The $Y_{\mathrm{h}}$-axis of excitation was also investigated. The results are presented separately for the palm and the fingers in Fig. 8.

\subsection{Apparent Mass Distribution at the Hand}

This section presents the result of the $Z_{\mathrm{h}}$-axis excitation and the $Z_{\mathrm{h}}$-axis AM distribution, only. However, other axes could be researched similarly, as discussed in the following.

The AM distribution at the fingers $R_{\text {Fingers }, Z h}$ (Eq. (12)) and the palm $R_{\text {Palm,Zh }}$ (Eq. (15)) are shown in Figs. 9 and 10. If the upper and lower sides of the fingers/palm were equally loaded the AM distribution and would be close to 1. However, from Figs. 9 and 10 it is clear that this is not the case. The are two distinct frequency regions (20 to $40 \mathrm{~Hz}$ and 80 to 100 $\mathrm{Hz}$ ) where the upper fingers take up to $45 \%$ more load. At frequencies close to $10 \mathrm{~Hz}$ the measurements show that more load is taken by the lower fingers.

The palm AM distribution shows a different trend: when the excitation is in the frequency range 10 to $60 \mathrm{~Hz}$ the lower palm side takes more load; however, from $60 \mathrm{~Hz}$ onward, the upper side of the palm is loaded more (up to $80 \%$ ). Above approximately $200 \mathrm{~Hz}$ the AM distribution is close to 1 for the finger as well as for the palm side.

\section{CONCLUSIONS}

A novel handle for assessing the biodynamic characteristics of a human hand-arm system has been developed. The use of a hand-held adapter is not required and, in addition to the palm, measurements of the fingers can be made concurrently. All of the sensors are located inside the handle; therefore, a force plate or a force sensor at the shaker-handle interface, used for measuring the push force, are not required. Since the handle is fitted with 3 -axes sensors, it is capable of measurements in all three hand directions $\left(Z_{\mathrm{h}}, Y_{\mathrm{h}}, X_{\mathrm{h}}\right)$.

The results of the AM for the bare hand are presented for the $Z_{\mathrm{h}}$ - and $Y_{\mathrm{h}}$-axis of excitation. The results matched with those observed by other researchers. The distribution of the apparent mass of the hand was also researched to examine the load on different parts of the hand. The results confirmed that the hand is not uniformly loaded, as the apparent masses were found to vary by up to $200 \mathrm{~Hz}$. The thumb, index, and middle fingers, on average, exert more force on the handle than the ring and the pinky finger, resulting in a higher apparent mass. The palm side has a mixed trend: the lower part of the palm produces a higher apparent

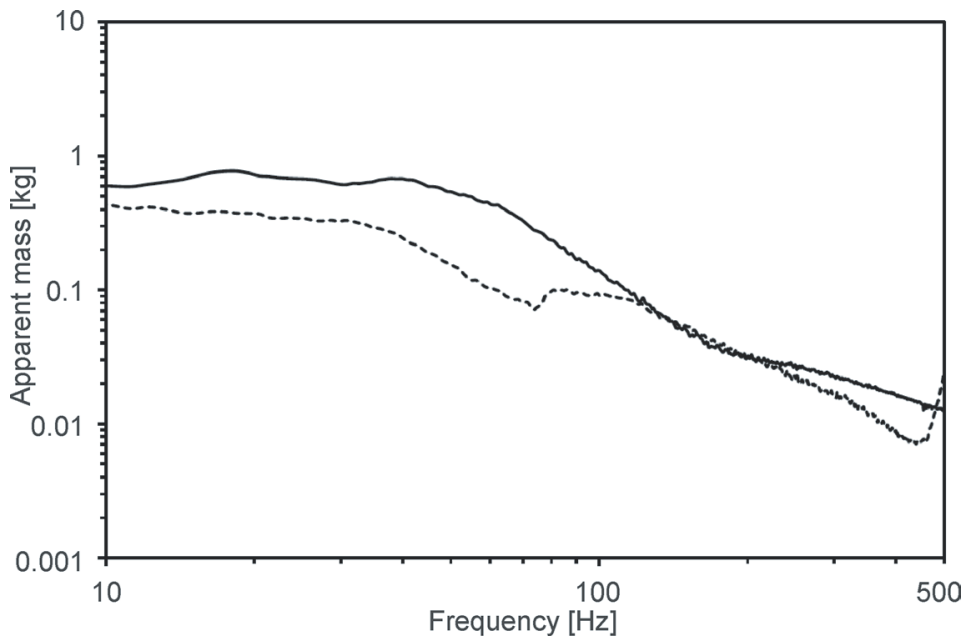

Fig. 8. Apparent mass of the subject for $Y_{h}$-axis excitation (- palm; - - - fingers) 


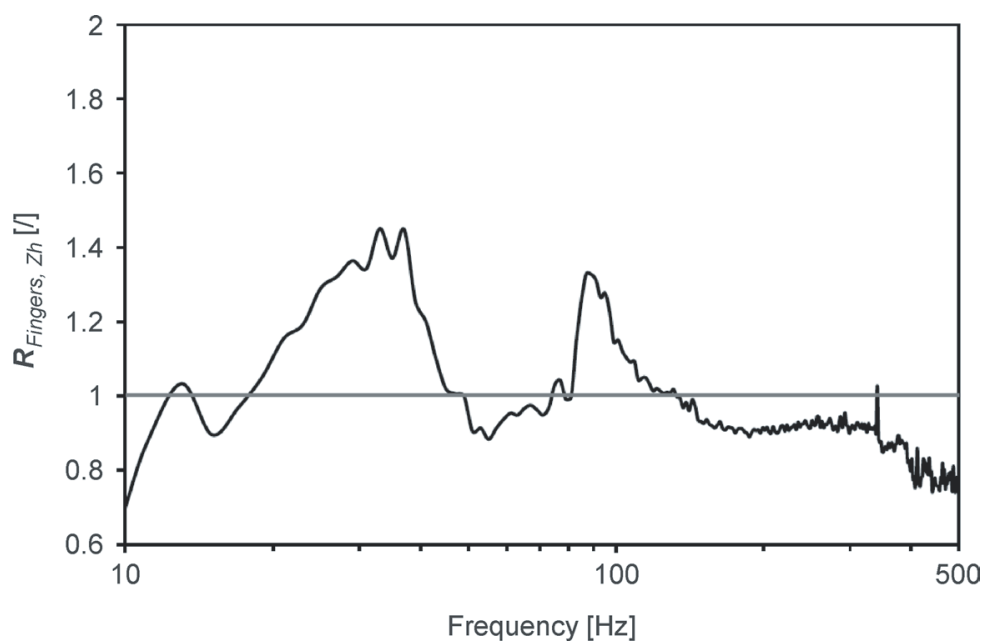

Fig. 9. Apparent mass relations between the upper and lower parts of the hand (fingers side)

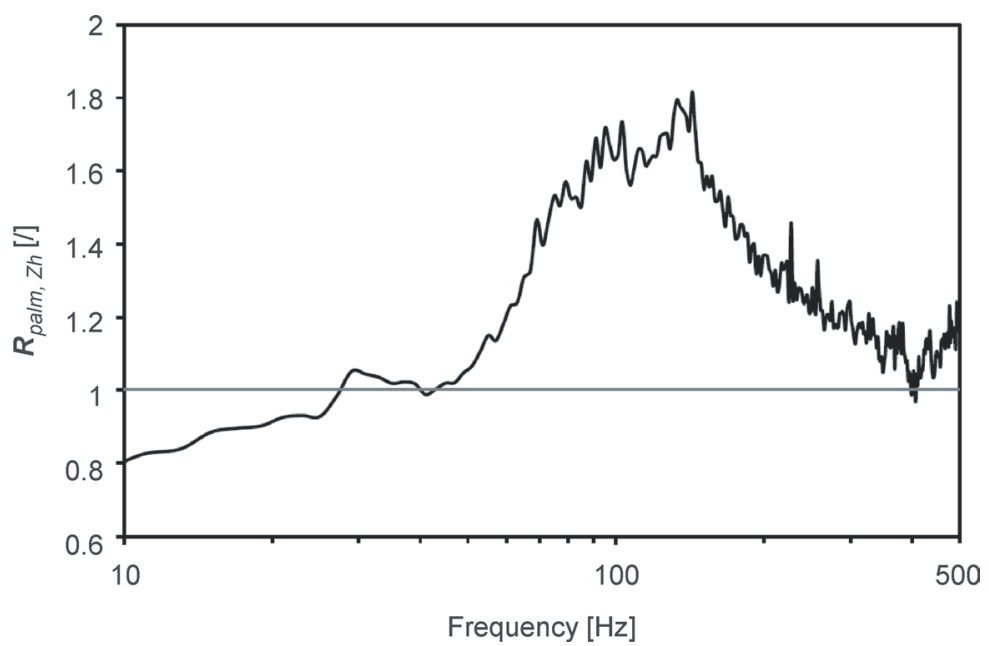

Fig. 10. Apparent mass relations between the upper and lower parts of the hand (palm side)

mass below $60 \mathrm{~Hz}$, but at higher frequencies the trend changes and the upper part is more loaded.

\section{REFERENCES}

[1] Griffin, M.J. (1990). Handbook of Human Vibration. Academic Press, London.

[2] ISO-10819:1996. Mechanical Vibration and ShockHand-Arm Vibration-Method for the Measurement and Evaluation of the Vibration Transmissibility of Gloves at the Palm of the Hand. International Organization for Standardization, Geneva.

[3] Dong, R.G., Welcome, D.E., McDowell, T.W., Wu, J.Z. (2006). Measurement of biodynamic response of human hand-arm system. Journal of Sound and Vibration, vol. 294, no. 4-5, p. 807-827, DOI:10.1016/j. jsv.2005.12.047.

[4] Shibata, N., Hosoya, N., Maeda, S., (2008). Establishment of one-axis vibration test system for measurement of biodynamic response of human handarm system. Industrial Health, vol. 46, no. 6, p. 629634, DOI:10.2486/indhealth.46.629.

[5] Dong, R.G., Welcome, D.E., McDowell, T.W., Wu, J.Z. (2009). Methods for deriving a representative biodynamic response of the hand-arm system to vibration. Journal of Sound and Vibration, vol. 325, no. 4-5, p. 1047-1061, DOI:10.1016/j.jsv.2009.04.006.

[6] Dong, R.G., Rakheja, S., McDowell, T.W., Welcome, D.E., Wu, J.Z., Warren, C., Barkley, J., Washington, B., Schopper, A.W. (2005). A method for assessing the effectiveness of anti-vibration gloves using biodynamic responses of the hand-arm system. Journal of Sound and Vibration, vol. 282, no. 3-5, p. 1101-1118, DOI:10.1016/j.jsv.2004.03.069.

[7] Dong, R.G., Wu, J.Z., Welcome, D.E. (2005). Recent advances in biodynamics of human hand-arm system. Industrial Health, vol. 43, no. 3, p. 449-471, DOI:10.2486/indhealth.43.449. 
[8] Concettoni, E., Griffin, M. (2009). The apparent mass and mechanical impedance of the hand and the transmission of vibration to the fingers, hand, and arm. Journal of Sound and Vibration, vol. 325, no. 3, p. 664678, DOI:10.1016/j.jsv.2009.03.033.

[9] Dong, R.G., McDowell, T.W., Welcome, D.E., Warren, C., Wu, J.Z., Rakheja, S. (2009). Analysis of antivibration gloves mechanism and evaluation methods. Journal of Sound and Vibration, vol. 321, no. 1-2, p. 435-453, DOI:10.1016/j.jsv.2008.09.044.

[10] ISO-5349-1:2001. Mechanical Vibration Measurement and Evaluation of Human Exposure to the Hand-Transmitted Vibration - Part 1: General Requirements. International Organization for Standardization, Geneva.

[11] ISO-10068:1998. Mechanical Vibration and ShockFree, Mechanical Impedance of the Human Hand-Arm System at the Driving Point. International Organization for Standardization, Geneva.

[12] Dong, R.G., Welcome, D.E., McDowell, T.W., Wu, J.Z., Schopper, A.W. (2006). Frequency weighting derived from power absorption of fingers-hand-arm system under $\mathrm{z}(\mathrm{h})$-axis vibration. Journal of Biomechanics, vol. 39, no. 12, p. 2311-2324, DOI:10.1016/j. jbiomech.2005.07.028.

[13] Stelling, J., Dupuis, H. (1996). Different acute effects of single-axis and multi-axis hand-arm vibration. International Archives of Occupational and Enviromental Health, vol. 68, no. 4, p. 236-242, DOI:10.1007/BF00381434.

[14] Dong, R.G., Welcome, D.E., Xu, X.S., Warren, C., McDowell, T.W., Wu, J.Z., Rakheja, S. (2012). Mechanical impedances distributed at the fingers and palm of the human hand in three orthogonal directions.
Journal of Sound and Vibration, vol. 331, no. 5, p. 1191-1206, DOI:10.1016/j.jsv.2011.10.015.

[15] Knez, L., Slavič, J., Boltežar, M. (2011). Vibration transmissibilities of the human hand arm system exposed to $\mathrm{Zh}$ - and $\mathrm{Yh}$ - axes vibration. Proceedings of the Kuhljevi dnevi conference, p. 97-104.

[16] Adewusi, S.A., Rakheja, S., Marcotte, P., Boileau, P.E. (2008). On the discrepancies in the reported human hand-arm impedance at higher frequencies. International Journal of Industrial Ergonomics, vol. 38, no. 9-10, p. 703-714, DOI:10.1016/j. ergon.2007.12.004.

[17] Ewinsm, D.J. (2000). Modal Testing Theory, Practice and Application - Second Edition. Research studies press ltd., Baldock, Hertfordshire.

[18] Maia, N.N.M., Silva, J.M.M. (1997). Theoretical and Experimental Modal Analysis. Research studies press ltd., Taunton, Somerset.

[19] Česnik, M., Slavič, J., Boltežar, M. (2009). Spatialmode-shape identification using a continuous wavelet transform. Strojniški vestnik - Journal of Mechanical Engineering, vol. 55, no. 5, p. 277-285.

[20] Aldien, Y., Marcotte, P., Rakheja, S., Boileau, P.E. (2006). Influence of hand-arm posture on biodynamic response of the human hand-arm exposed to $\mathrm{z}(\mathrm{h})$ axis vibration. International Journal of Industrial Ergonomics, vol. 36, no. 1, p. 45-59, DOI:10.1016/j. ergon.2005.07.001.

[21] Adewusi, S.A., Rakheja, S., Marcotte, P., Boution, J. (2010). Vibration transmissibility characteristics of the human hand-arm system under different postures, hand forces and excitation levels. Journal of Sound and Vibration, vol. 329, no. 14, p. 2953-2971, DOI:10.1016/j.jsv.2010.02.001. 